TRANSACTIONS OF THE

AMERICAN MATHEMATICAL SOCIETY

Volume 354, Number 6, Pages 2475-2494

S 0002-9947(02)02961-6

Article electronically published on February 1, 2002

\title{
TOPOLOGICAL DYNAMICS ON MODULI SPACES II
}

\author{
JOSEPH P. PREVITE AND EUGENE Z. XIA
}

\begin{abstract}
Let $M$ be an orientable genus $g>0$ surface with boundary $\partial M$. Let $\Gamma$ be the mapping class group of $M$ fixing $\partial M$. The group $\Gamma$ acts on $\mathcal{M}_{\mathcal{C}}=\operatorname{Hom}_{\mathcal{C}}\left(\pi_{1}(M), \mathrm{SU}(2)\right) / \mathrm{SU}(2)$, the space of $\mathrm{SU}(2)$-gauge equivalence classes of flat $\mathrm{SU}(2)$-connections on $M$ with fixed holonomy on $\partial M$. We study the topological dynamics of the $\Gamma$-action and give conditions for the individual $\Gamma$-orbits to be dense in $\mathcal{M}_{\mathcal{C}}$.
\end{abstract}

\section{INTRODUCTION}

Let $M$ be an orientable surface of genus $g$ with $n$ boundary components (circles). Let

$$
\left\{C_{1}, C_{2}, \ldots, C_{n}\right\} \subset \pi_{1}(M)
$$

be elements in the fundamental group that correspond to these $n$ boundary components.

The representation space $\operatorname{Hom}\left(\pi_{1}(M), \mathrm{SU}(2)\right)$ has a natural topology [4] 5]. The group $\mathrm{SU}(2)$ acts on $\operatorname{Hom}\left(\pi_{1}(M), \mathrm{SU}(2)\right)$ by conjugation. Define the resulting quotient space to be

$$
\mathcal{M}=\operatorname{Hom}\left(\pi_{1}(M), \mathrm{SU}(2)\right) / \mathrm{SU}(2) \text {. }
$$

A conjugacy class in $\mathrm{SU}(2)$ is determined by its trace in $[-2,2]$. To each $C_{i}$, assign a trace $-2 \leq c_{i} \leq 2$ and let

$$
\mathcal{C}=\left\{c_{1}, c_{2}, \ldots, c_{n}\right\}
$$

Definition 1.1. The moduli space with fixed holonomy $\mathcal{C}$ is

$$
\mathcal{M}_{\mathcal{C}}=\left\{[\rho] \in \mathcal{M}: \operatorname{tr}\left(\rho\left(C_{i}\right)\right)=c_{i}, 1 \leq i \leq n\right\} .
$$

The space $\mathcal{M}_{\mathcal{C}}$ is compact, but possibly singular. The set of smooth points of $\mathcal{M}_{\mathcal{C}}$ possesses a natural symplectic structure which gives rise to a finite measure $\mu$ on $\mathcal{M}_{\mathcal{C}}$ (see [4, 5]).

Let $\operatorname{Diff}(M, \partial M)$ be the group of diffeomorphisms fixing $\partial M$. The mapping class group $\Gamma$ is defined to be $\pi_{0}(\operatorname{Diff}(M, \partial M))$. The group $\Gamma$ acts on $\pi_{1}(M)$. This action induces an action on $\operatorname{Hom}\left(\pi_{1}(M), \mathrm{SU}(2)\right): \Gamma \times \operatorname{Hom}\left(\pi_{1}(M), \mathrm{SU}(2)\right) \longrightarrow$ $\operatorname{Hom}\left(\pi_{1}(M), \operatorname{SU}(2)\right)$. If $\gamma \in \Gamma$ and $\rho \in \operatorname{Hom}\left(\pi_{1}(M), \operatorname{SU}(2)\right)$, then $(\gamma \circ \rho)(X)=$ $\rho\left(\gamma^{-1}(X)\right)$. This, in turn, gives an action $\Gamma \times \mathcal{M}_{\mathcal{C}} \longrightarrow \mathcal{M}_{\mathcal{C}}$.

Theorem 1.2 (Goldman [4]). The mapping class group $\Gamma$ acts ergodically on $\mathcal{M}_{\mathcal{C}}$.

Received by the editors September 26, 2000 and, in revised form, June 28, 2001.

2000 Mathematics Subject Classification. Primary 57M05, 54H20.

Key words and phrases. Fundamental group of a surface, mapping class group, Dehn twist, topological dynamics, moduli spaces. 
One may also study the topological dynamics of the mapping class group action on $\mathcal{M}_{\mathcal{C}}$. The topological-dynamical problem is considerably more delicate. To begin with, not all orbits are dense in $\mathcal{M}_{\mathcal{C}}$. If $G$ is a proper closed subgroup of $\mathrm{SU}(2)$, then the image of $\operatorname{Hom}\left(\pi_{1}(M), G\right)$ in $\mathcal{M}_{\mathcal{C}}$ is proper, closed and $\Gamma$-invariant. The case of the one-holed torus has been dealt with in $[9$ :

Theorem 1.3. Suppose $M$ is a torus with one boundary component, and that $\rho \in$ $\operatorname{Hom}\left(\pi_{1}(M), \mathrm{SU}(2)\right)$ is such that $\rho\left(\pi_{1}(M)\right)$ is dense in $\mathrm{SU}(2)$. Then the $\Gamma$-orbit of the conjugacy class $[\rho] \in \mathcal{M}_{\mathcal{C}}$ is dense in $\mathcal{M}_{\mathcal{C}}$.

This paper deals with the general case of $g>0$ and proves:

Theorem 1.4. Suppose $M$ is an orientable surface with boundary having genus greater than zero. Let $\rho \in \operatorname{Hom}\left(\pi_{1}(M), \mathrm{SU}(2)\right)$ be such that $\rho\left(\pi_{1}(M)\right)$ is dense in $\mathrm{SU}(2)$. Then the $\Gamma$-orbit of the conjugacy class $[\rho] \in \mathcal{M}_{\mathcal{C}}$ is dense in $\mathcal{M}_{\mathcal{C}}$.

The group $\mathrm{SU}(2)$ double covers the group $\mathrm{SO}(3)$. The group $\mathrm{SO}(3)$ contains closed subgroups isomorphic to $\mathrm{O}(2)$, as well as the symmetry groups of the regular polyhedra: $T^{\prime}$ (the tetrahedron), $C^{\prime}$ (the cube), $D^{\prime}$ (the dodecahedron), and their subgroups. The inverse images (by the covering map) of $\mathrm{O}(2), T^{\prime}, C^{\prime}, D^{\prime}$ will be called $\operatorname{Pin}(2), T, C, D$, respectively. The identity component of $\operatorname{Pin}(2)$ is called $\operatorname{Spin}(2)$. Let $\rho \in \operatorname{Hom}\left(\pi_{1}(M), \mathrm{SU}(2)\right)$. Theorem 1.4 implies that if $\rho\left(\pi_{1}(M)\right)$ is not contained in a group isomorphic to $C, D$, or $\operatorname{Pin}(2)$, then the $\Gamma$-orbit of the conjugacy class $[\rho] \in \mathcal{M}_{\mathcal{C}}$ is dense in $\mathcal{M}_{\mathcal{C}}$. Theorem 1.4 covers all moduli spaces except those of the $n$-holed spheres.

1.1. Outline of the proof. A pants decomposition $\mathcal{P}$ of $M$ gives rise to a smooth open dense subset $\mathcal{M}_{\mathcal{P}}$ that is an integrable system (see [7]) inside the moduli space $\mathcal{M}_{\mathcal{C}}$. Hence, one obtains the following diagram [4]:

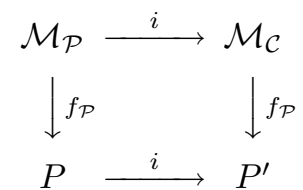

where $\mathcal{M}_{\mathcal{P}}$ is a torus bundle over $P$, and $P^{\prime} \subset[-2,2]^{N}$, where $N=\frac{1}{2} \operatorname{dim}\left(\mathcal{M}_{\mathcal{C}}\right)$. The subgroup $\Gamma_{\mathcal{P}} \subset \Gamma$ that preserves the fibres of $f_{\mathcal{P}}$ acts as rotations on each fibre with angles depending on the base coordinates [4. Section 2 gives a brief outline of this integrable system and the decomposition of the $\Gamma$-action.

The proof of Theorem 1.4 involves two steps. Suppose that $[\rho]$ is a generic representation (i.e. $\rho\left(\pi_{1}(M)\right)$ is dense in $\left.\mathrm{SU}(2)\right)$. Let $\Gamma([\rho])$ denote the $\Gamma$-orbit of $[\rho]$. The first step is to show that if $f_{\mathcal{P}}(\Gamma([\rho]))$ is dense in $P$, then $\Gamma([\rho])$ is dense in $\mathcal{M}_{\mathcal{C}}$ (Corollary 2.4). The second step involves proving the base density theorem, i.e., the density of $f_{\mathcal{P}}(\Gamma([\rho]))$ in $P$.

As the problem deals with arbitrary genus, the proof involves induction with the one-holed torus as the base case. For a generic representation $\rho$, one first shows that there is a one-holed torus $T$ inside $M$ such that the restriction of $\rho$ to $\pi_{1}(T)$ is generic. This is a detailed combinatorial calculation which is outlined in Section 3

After obtaining a generic handle, we proceed to demonstrate the base density theorem for the $(n+2 g-2)$-holed torus. An analysis of the case of the four-holed sphere is required to get the induction process started. This is used to prove the result for the case of the two-holed torus. From there, the case of the three-holed 
torus is proven, which, in turn, is used to prove the case of the $(n+2 g-2)$-holed torus.

To complete the proof, the $2 g-2$ holes of the $(n+2 g-2)$-holed torus are grouped in pairs, and each pair is glued along their boundary to obtain the original surface $M$ with genus $g$ and $n$ boundary components.

1.2. Some definitions. Fix a surface $M$ with genus $g>0$ and $n$ boundary components. Then $M$ may be described as an $n$-holed $2 g$-gon, with appropriate identifications. More precisely, the fundamental group $\pi_{1}(M, O)$ is generated by $S=\left\{A_{i}\right\}_{i=1}^{2 g+n}$, subject to the relation

\section{Definition 1.5.}

$$
\left(\prod_{i=1}^{g}\left[A_{i}, A_{i+g}\right]\right)\left(\prod_{i=2 g+1}^{2 g+n} A_{i}\right)=e .
$$

1. A representation $\rho$ into $\mathrm{SU}(2)$ is generic if the image of $\rho$ is dense in $\mathrm{SU}(2)$.

2. A handle $(A, B)$ consists of two simple loops $A, B \in \pi_{1}(M, O)$ crossing at $O$, but otherwise disjoint (see [3]).

3. A handle $(A, B)$ is generic with respect to a representation $\rho$ if the image of $\langle A, B\rangle \subset \pi_{1}(M)$ is dense in $\mathrm{SU}(2)$ (cf. Theorem 1.3).

4. Suppose $G \subset \mathrm{SU}(2)$. A representation $\rho$ is said (resp. not) to be $G$ if $\operatorname{Im}(\rho)$ is (resp. not) contained in some (resp. any) isomorphic copy of $G$ in $\mathrm{SU}(2)$.

5. Associated to each simple loop $A \in \pi_{1}(M, O)$ is the Dehn twist in $A$, represented in $\Gamma$ by a diffeomorphism of $M$. The action of the Dehn twist amounts to cutting $M$ at $A$, twisting one of the resulting boundary circles once, and then re-identifying the two circles.

6. With a fixed representation $\rho, X \in \pi_{1}(M, O)$, and $\gamma \in \Gamma$, we write $X$ for $\rho(X)$ and $\gamma(X)$ for $\gamma(\rho)(X)$ when there is no ambiguity. A small letter will be used to denote the trace of the matrix represented by the corresponding capital letter. For example, we use $x$ to denote $\operatorname{tr}(\rho(X))$. In this setting, $\gamma(x)$ denotes $\operatorname{tr}(\gamma(\rho)(X))$.

7. Let $\langle V, \mathrm{~d}\rangle$ be a metric space. For any $\epsilon>0$, a subset $U \subset V$ is $\epsilon$-dense in $V$ if for each $v \in V$, there exists a point $u \in U$ such that $\mathrm{d}(u, v)<\epsilon$.

Acknowledgments. The authors thank Paul Becker, Richard Stankewitz, and the referee for their helpful comments. Eugene Xia was at the University of Arizona when much of this research was carried out. He also thanks IHÉS for its hospitality. We also mention here that William Goldman has communicated to us that Michael Kapovich recently obtained similar results.

\section{Moduli Spaces as Tori Bundles}

We begin by giving a brief description of the integrable system on the moduli space [5]. Suppose $M$ has genus $g \geq 1$ and $n \geq 0$ boundary components. Let

$$
\mathcal{C}=\left\{c_{1}, \ldots, c_{n}\right\}
$$

be a fixed set of conjugacy classes with $c_{i} \neq \pm 2$ for all $i$. Then the real dimension of $\mathcal{M}_{\mathcal{C}}$ is $6 g-6+2 n$. Since the case of the torus is well understood [9], we assume throughout the remainder of the paper that $g>1$ or $n>0$.

There is a map

$$
f_{\mathcal{P}}: \mathcal{M}_{\mathcal{C}} \longrightarrow[-2,2]^{N}
$$


that arises from a pants decomposition $\mathcal{P}$ of $M$, where $N=3 g-3+n$ (see 4]). Fix a pants decomposition $\mathcal{P}$ of $M$. This provides $3 g-3+n$ loops $B_{1}, \ldots, B_{3 g-3+n} \in$ $\pi_{1}(M, O)$. Let $[\rho] \in \mathcal{M}_{\mathcal{C}}$ be given. Then

$$
f_{\mathcal{P}}([\rho])=\left(b_{1}, \ldots, b_{3 g-3+n}\right)
$$

is the desired map, where $b_{i}=\operatorname{tr}\left(\rho\left(B_{i}\right)\right)$. Let $\beta=\left(b_{1}, \ldots, b_{3 g-3+n}\right)$, let $P^{\prime}$ be the image of $f_{\mathcal{P}}$ and let $P=P^{\prime} \backslash \partial P^{\prime}$. The map $f_{\mathcal{P}}$ restricted to $\mathcal{M}_{\mathcal{P}}=f_{\mathcal{P}}^{-1}(P)$ is a submersion [4]. Denote by $\Gamma_{\mathcal{P}} \subset \Gamma$ the stabilizer of the fibres of $f_{\mathcal{P}}$.

Proposition 2.1. The set $P^{\prime}$ consists of all $\beta \in[-2,2]^{3 g-3+n}$ that simultaneously satisfy the $2 g-2+n-1$ inequalities

$$
b_{i}^{2}+b_{j}^{2}+b_{k}^{2}-b_{i} b_{j} b_{k} \leq 4,
$$

where the three curves $B_{i}, B_{j}$ and $B_{k}$ bound a triply punctured sphere in the decomposition $\mathcal{P}$ of $M$ (this includes the possibility of $B_{i}$ being a boundary curve in $\partial M)$. Moreover, for $\beta \in P$, there is a $\Gamma_{\mathcal{P}}$-equivariant homeomorphism

$$
h: f_{\mathcal{P}}^{-1}(\beta) \rightarrow T^{3 g-3+n}
$$

such that for all $\xi \in f_{\mathcal{P}}^{-1}(\beta)$ and $\left(n_{1}, \ldots, n_{3 g-3+n}\right) \in \mathbb{Z}^{3 g-3+n}$

$$
h: \tau_{1}^{n_{1}} \ldots \tau_{3 g-3+n}^{n_{3 g-3+n}} \xi \mapsto\left[\begin{array}{c}
e^{i n_{1} \theta_{1}} h_{1} \\
\vdots \\
e^{i n_{3 g-3+n} \theta_{3 g-3+n}} h_{3 g-3+n}
\end{array}\right],
$$

where

$$
h(\xi)=\left[\begin{array}{c}
h_{1} \\
\vdots \\
h_{3 g-3+n}
\end{array}\right]
$$

$\theta_{j}=\cos ^{-1}\left(b_{j} / 2\right)$, and $\tau_{i}$ is the action of the Dehn twist in $B_{i}$.

Proof. See 4].

In short, $\left(\mathcal{M}_{\mathcal{P}}, f_{\mathcal{P}}\right)$ is an integrable system (see [7] for details). The real dimensions of $P$ and $\mathcal{M}_{\mathcal{P}}$ are $3 g-3+n$ and $6 g-6+2 n$, respectively. Denote by $\mathcal{M}_{\mathcal{C}}^{s}$ the subset of irreducible representations of $\mathcal{M}_{\mathcal{C}}$.

Lemma 2.2. $\mathcal{M}_{\mathcal{C}}^{s}$ is smooth, open, and dense in $\mathcal{M}_{\mathcal{C}}$.

Proof. See [4, 5].

Proposition 2.3. The subset $\mathcal{M}_{\mathcal{P}}$ is open and dense in $\mathcal{M}_{\mathcal{C}}^{s}$.

Proof. A direct calculation from Proposition 2.1 shows that $\mathcal{M}_{\mathcal{C}}^{s} \backslash \mathcal{M}_{\mathcal{P}}$ is a real algebraic subvariety with positive codimension. The result then follows from the fact that $\mathcal{M}_{\mathcal{C}}^{s}$ is smooth and has dimension $6 g-6+2 n$.

Together, Propositions 2.1 and 2.3 imply:

Corollary 2.4. Let $[\rho] \in \mathcal{M}_{\mathcal{C}}$, and let $\Gamma([\rho])$ be the $\Gamma$-orbit of $[\rho]$. If $f_{\mathcal{P}}(\Gamma([\rho]))$ is dense in $P$, then $\Gamma([\rho])$ is dense in $\mathcal{M}_{\mathcal{C}}$. 


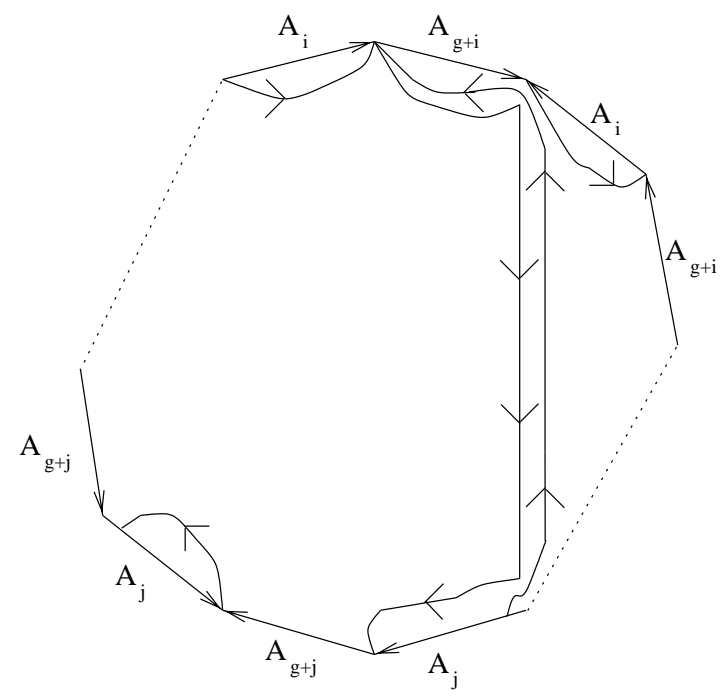

Figure $1 .\left(A_{i}, A_{g+i} A_{j}\right)$ is a handle.

\section{Generic Representations and Handles}

Here we adapt an idea in $[3$ to find a generic handle (one-holed torus) inside $M$.

Proposition 3.1. For any generic $\rho \in \operatorname{Hom}\left(\pi_{1}(M, O), \mathrm{SU}(2)\right)$, there exists a generic handle $(A, B)$.

The proof of Proposition 3.1 is highly computational. One should first consult Section [5 and [9], as the proof involves the moduli spaces of one-holed tori and uses many ideas pertaining to those spaces.

The proof starts with a generic representation $\rho$, which when restricted to each handle $\left\langle A_{i}, A_{g+i}\right\rangle$ is $\operatorname{Spin}(2)$, but not $\operatorname{Spin}(2)$ on $M$. One shows that there exists $j$ such that $\left\langle A_{i}, A_{g+i} A_{j}\right\rangle$ is not $\operatorname{Spin}(2)$, with $\left\langle A_{i}, A_{g+i} A_{j}\right\rangle$ forming a handle (see Figure 1).

From there, one applies the same technique to the groups $\operatorname{Pin}(2), T, C$, and finally to $D$. This process amounts to numerous routine computations, which we omit here but which are available at http://vortex.bd.psu.edu/ jpp/td2/ in the form of MAPLE worksheets with accompanying text.

\section{The Three-Holed Sphere}

Suppose $M$ is a three-holed sphere. Then $\pi_{1}(M)$ has a presentation:

$$
\langle A, B, C: A B C=I\rangle,
$$

where $A, B$, and $C$ represent the homotopy classes of the three boundaries of $M$. A direct computation, together with results from [4] and [9], shows

\section{Proposition 4.1.}

1. A representation $\rho$ on a three-holed sphere is a $\operatorname{Spin}(2)$-representation if and only if $a^{2}+b^{2}+c^{2}-a b c-4=0$. 
2. A representation $\rho$ on a three-holed sphere is $\operatorname{Pin}(2)$ and not $\operatorname{Spin}(2)$ if and only if $a^{2}+b^{2}+c^{2}-a b c-4 \neq 0$ and at least two of the three values $a, b, c$ are zero.

\section{The One-Holed Torus}

We briefly summarize some relevant results that appear in [4] and 9. Suppose that $M$ is a one-holed torus. The fundamental group $\pi_{1}(M)$ has a presentation

$$
\pi_{1}(M)=\left\langle X, Y, K: K=X Y X^{-1} Y^{-1}\right\rangle,
$$

where $K$ represents the element corresponding to the boundary component. Let

$$
E=\operatorname{Hom}\left(\pi_{1}(M), \mathrm{SU}(2)\right) / \mathrm{SU}(2) .
$$

A representation class $[\rho] \in E$ is determined by

$$
x=\operatorname{tr}(\rho(X)), \quad y=\operatorname{tr}(\rho(Y)), \quad z=\operatorname{tr}(\rho(X Y)) .
$$

Therefore, there is a global coordinate chart:

$$
[\rho] \stackrel{F}{\longmapsto}(x, y, z) .
$$

In addition, $k=\operatorname{tr}(\rho(K))$ is given by the formula

$$
k=\operatorname{tr}(\rho(K))=x^{2}+y^{2}+z^{2}-x y z-2 .
$$

Let

$$
E_{k}=\left\{(x, y, z) \in[-2,2]^{3}: x^{2}+y^{2}+z^{2}-x y z-2=k\right\}
$$

then

$$
E=\bigcup_{k \in[-2,2]} E_{k}
$$

For $-2<k<2$, the set $E_{k}$ is a smooth two-sphere; the set $E_{2}$ is a singular sphere, and $E_{-2}=(0,0,0)$.

The mapping class group $\Gamma$ is generated by the maps $\tau_{X}$ and $\tau_{Y}$ induced by the Dehn twists in $X, Y \in \pi_{1}(M)$. These act on the fundamental group as

$$
\tau_{X}(X)=X \text { and } \tau_{X}(Y)=Y X^{-1}
$$

and

$$
\tau_{Y}(X)=X Y^{-1} \text { and } \tau_{Y}(Y)=Y .
$$

The effect on a representation $\rho$ is as follows:

$$
\left(\tau_{X} \circ \rho\right)(X)=\rho(X) \text { and } \tau_{X}(\rho(Y))=\rho(Y X)
$$

and

$$
\left(\tau_{Y} \circ \rho\right)(X)=\rho(X Y) \text { and } \tau_{Y}(\rho(Y))=\rho(Y) .
$$

Remark 5.1. The reader should be aware that when $Y$ actually stands for $\rho(Y)$ (Definition 1.56), $\tau_{X}(Y)=Y X$.

The induced action of $\Gamma$ on $E$ preserves $E_{k}$. The actions can be described explicitly [4]:

$$
\begin{aligned}
& \tau_{X}(x, y, z)=(x, z, x z-y), \\
& \tau_{Y}(x, y, z)=(z, y, y z-x) .
\end{aligned}
$$


The action of $\tau_{X}$ fixes $x$ and $k$, and preserves the ellipse

$$
X_{k}(x)=\{x\} \times\left\{(y, z): \frac{2-x}{4}(y+z)^{2}+\frac{2+x}{4}(y-z)^{2}=2+k-x^{2}\right\} .
$$

A change of coordinates transforms $X_{k}(x)$ into the circle

$$
X_{k}(x)=\{x\} \times\left\{(\tilde{y}, \tilde{z}): \tilde{y}^{2}+\tilde{z}^{2}=2+k-x^{2}\right\}
$$

(see [4, 9]). In this new coordinate system, $\tau_{X}$ acts as a rotation by $\cos ^{-1}(x / 2)$. In short, the sphere $E_{k}$ is the union of circles

$$
E_{k}=\bigcup_{x} X_{k}(x)
$$

and $\tau_{X}$ rotates (up to a coordinate transformation) each level set $X_{k}(x)$ by an angle of $\cos ^{-1}(x / 2)$. Similarly, there is a coordinate transformation so that $\tau_{Y}$ acts as a rotation of $Y_{k}(y)$ by an angle of $\cos ^{-1}(y / 2)$.

Proposition 5.2. The space of $\operatorname{Spin}(2)$ representation classes consists precisely of $E_{2}$. The Pin(2) representation classes consist of $E_{2}$ and the intersections of the three coordinate axes with $E$. For each $-2<k<2$, there are exactly six points corresponding to $\operatorname{Pin}(2)$ representation classes in $E_{k}$. Moreover, a representation class $(x, y, z) \in E_{k}$ with $-2<k<2$ and $x \neq 0$ is $\operatorname{Pin}(2)$ if and only if $k=x^{2}-2$.

Proof. See [9].

Remark 5.3. This is the first explicit example of Proposition 2.1 with $g=1$ and $n=1$. One obtains a pair of pants by cutting along $X$ (resp. $Y$ ). The important property is that if $X_{k}(x)$ (resp. $Y_{k}(y)$ ) is a non-degenerate circle, then $\tau_{X}$ (resp. $\tau_{Y}$ ) acts on the fibre $X_{k}(x)$ (resp. $Y_{k}(y)$ ) as a rotation with an angle depending solely on $x$ (resp. $y$ ) and is independent of either $k$ or $y$ (resp. $x$ ). In particular, if a representation $\rho$ is not $\operatorname{Pin}(2)$, then neither $\tau_{X}$ nor $\tau_{Y}$ fixes $[\rho]$.

Remark 5.4. Let $\rho$ be generic. By Theorem 1.3 , the $\left\langle\tau_{X}, \tau_{Y}\right\rangle$-orbit $\mathcal{O}$ of $[\rho]$ is dense in $E_{k}$. Hence, there is a number $r>0$ such that the set $\{(x, y):(x, y, z) \in \mathcal{O}\}$ is dense in $R=[-r, r]^{2}$. In particular, by Dehn twisting in $\tau_{X}$ and $\tau_{Y}$, one can always assume that $x$ and $y$ are simultaneously off of any finite set of values and are arbitrarily close to zero.

Remark 5.5. In addition to Theorem 1.3 it was shown in 9 that the $k$-values for the surjective $T, C, D$ representations are in the set

$$
\mathcal{S}=\left\{\frac{1 \pm \sqrt{5}}{2}, 0,1\right\}
$$

In particular, if $(x, y, z)$ is not $\operatorname{Pin}(2)$ and $k \notin \mathcal{S}$, then $(x, y, z)$ is generic.

\section{The Four-Holed Sphere}

We first review some results that appear in [1] and [4]. Suppose $M$ is a four-holed sphere. Then the fundamental group $\pi_{1}(M, O)$ admits a presentation

$$
\langle A, B, C, D: A B C D=I\rangle \text {. }
$$




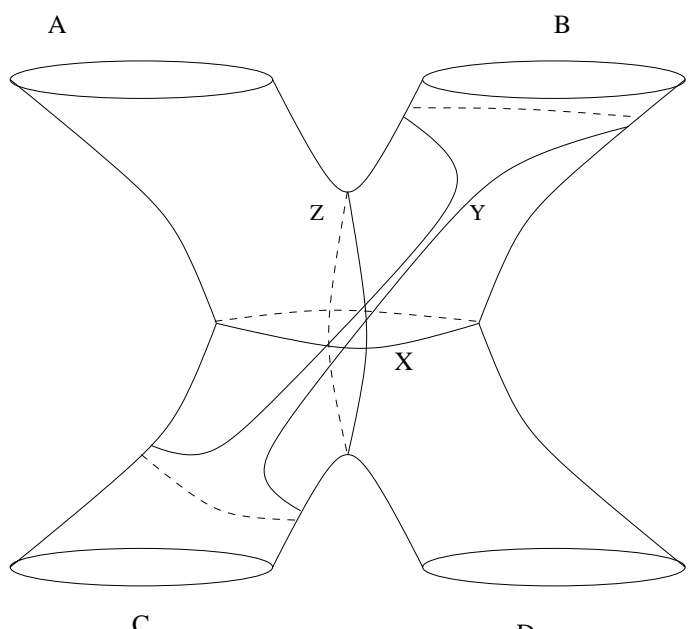

$\mathrm{C}$

$\mathrm{D}$

Figure 2. The four-holed sphere

6.1. The moduli space $\mathcal{M}$. If one of $a, b, c, d= \pm 2$, the moduli space can be identified with a moduli space of a three-holed sphere, so we assume that $a, b, c, d \neq$ \pm 2 . The moduli space $\mathcal{M}$ for the four-holed sphere is six-dimensional. Let $g$ : $\mathcal{M} \longrightarrow[-2,2]^{4}$ be the map defined by $g([\rho])=(a, b, c, d)$.

Set $X=A B, Y=B C$, and $Z=C A$ (see Figure 2). With the $(x, y, z)$-global coordinates, the moduli space $E_{(a, b, c, d)}=g^{-1}(a, b, c, d)$ is the level set defined by the equation

$$
\begin{aligned}
x^{2}+y^{2}+z^{2}+x y z= & (a b+c d) x+(a d+b c) y+(a c+b d) z \\
& -\left(a^{2}+b^{2}+c^{2}+d^{2}+a b c d-4\right) .
\end{aligned}
$$

Generically, $E_{(a, b, c, d)}$ is a topological two-sphere.

Let

$$
I_{a, b}=\left[\frac{a b-\sqrt{\left(a^{2}-4\right)\left(b^{2}-4\right)}}{2}, \frac{a b+\sqrt{\left(a^{2}-4\right)\left(b^{2}-4\right)}}{2}\right] .
$$

For any $\kappa=(a, b, c, d) \in(-2,2)^{4}$ with $x \in I_{a, b} \cap I_{c, d}$, the $x$-level sets $X_{\kappa}(x) \subset E_{\kappa}$ are ellipses (possibly degenerate) in $y$ and $z$ given by

$$
\begin{gathered}
\frac{2+x}{4}\left[(y+z)-\frac{(a+b)(d+c)}{2+x}\right]^{2}+\frac{2-x}{4}\left[(y-z)-\frac{(a-b)(d-c)}{2-x}\right]^{2} \\
=\frac{\left(x^{2}-a b x+a^{2}+b^{2}-4\right)\left(x^{2}-c d x+c^{2}+d^{2}-4\right)}{4-x^{2}} .
\end{gathered}
$$

If $x \notin I_{a, b} \cap I_{c, d}$, then the $x$-level sets are empty. There are similar descriptions for the $y$ - and $z$-level sets $Y_{\kappa}(y)$ and $Z_{\kappa}(z)$ respectively (see [4]).

For $x$ in the interior of $I_{a, b} \cap I_{c, d}$ the level set $X_{\kappa}(x)$ is an ellipse centered at

$$
\left\{\begin{array}{l}
y_{c}(x)=\frac{2[2(a d+b c)-x(a c+b d)]}{4-x^{2}}, \\
z_{c}(x)=\frac{2[2(a c+b d)-x(a d+b c)]}{4-x^{2}} .
\end{array}\right.
$$


Note that

$$
E_{\kappa}=\bigcup_{x \in I_{a, b} \cap I_{c, d}} X_{\kappa}(x)
$$

(see Proposition 2.1 and [1]). By symmetry, similar constructs exist for the $y$ - and $z$-coordinates.

6.2. The mapping class action. The Dehn twist along $X$ acts on $\pi_{1}(M)$ as follows:

$$
\tau_{X}(A)=A, \quad \tau_{X}(B)=B, \quad \tau_{X}(C)=X^{-1} C X, \quad \tau_{X}(D)=X^{-1} D X .
$$

This action effects the representation $\rho$ as

$$
\begin{gathered}
\tau_{X}(\rho(A))=\rho(A), \quad \tau_{X}(\rho(B))=\rho(B), \quad \tau_{X}(\rho(C))=\rho\left(X C X^{-1}\right), \\
\tau_{X}(\rho(D))=\rho\left(X D X^{-1}\right) .
\end{gathered}
$$

We define $\tau_{Y}$ and $\tau_{Z}$ similarly. In local coordinates, this implies that the actions of $\tau_{X}, \tau_{Y}, \tau_{Z}$ are

$$
\begin{aligned}
& {\left[\begin{array}{l}
y \\
z
\end{array}\right] \stackrel{\tau_{X}}{\longmapsto}\left[\begin{array}{c}
a d+b c-x(a c+b d-x y-z)-y \\
a c+b d-x y-z
\end{array}\right],} \\
& {\left[\begin{array}{l}
z \\
x
\end{array}\right] \stackrel{\tau_{Y}}{\longmapsto}\left[\begin{array}{c}
b d+c a-y(b a+c d-y z-x)-z \\
b a+c d-y z-x
\end{array}\right],} \\
& {\left[\begin{array}{l}
x \\
y
\end{array}\right] \stackrel{\tau_{Z}}{\longmapsto}\left[\begin{array}{c}
c d+a b-z(c b+a d-z x-y)-x \\
c b+a d-z x-y
\end{array}\right] .}
\end{aligned}
$$

These actions preserve the ellipses $X_{\kappa}(x) \subset E_{\kappa}, Y_{\kappa}(y) \subset E_{\kappa}$, and $Z_{\kappa}(z) \subset E_{\kappa}$, respectively. After coordinate transformations, these are rotations by the angles $2 \cos ^{-1}(x / 2), 2 \cos ^{-1}(y / 2)$, and $2 \cos ^{-1}(z / 2)$, respectively [4].

Remark 6.1. This is the second explicit example of Proposition 2.1 with $g=0$ and $n=4$. One obtains two pairs of pants by cutting along $X$ (resp. $Y$ or $Z$ ). The important property is that if $X_{\kappa}(x)$ (resp. $Y_{\kappa}(y)$ or $Z_{\kappa}(z)$ ) is a non-degenerate circle, then $\tau_{X}$ (resp. $\tau_{Y}$ or $\tau_{Z}$ ) acts on the fibre $X_{\kappa}(x)\left(\right.$ resp. $Y_{\kappa}(x)$ or $\left.Z_{\kappa}(z)\right)$ as a rotation with an angle depending only on $x$ (resp. $y$ or $z$ ) and is independent of $\kappa$.

Let $\mathrm{d}$ be the metric on $\mathbb{R}^{3}$ given by

$$
\mathrm{d}\left(\left(x_{1}, y_{1}, z_{1}\right),\left(x_{2}, y_{2}, z_{2}\right)\right)=\max \left\{\left|x_{1}-x_{2}\right|,\left|y_{1}-y_{2}\right|,\left|z_{1}-z_{2}\right|\right\} .
$$

The metric $\mathrm{d}$ generates the usual topology on $\mathcal{M}_{\mathcal{C}}$. For a fixed $\kappa$, the coordinates provide an embedding of $E_{\kappa}$ into $\mathbb{R}^{3}$.

6.3. Filtration on the level sets. We introduce a filtration that is analogous to the one introduced in [9] for the one-holed torus. The Dehn twist $\tau_{Y}$ acts on the (transformed) subsets $Y_{\kappa}(y)$ via a rotation of angle $2 \cos ^{-1}(y / 2)$. Thus there is a filtration of the $y$-coordinates that yields finite orbits under $\tau_{Y}$ as follows:

Let $Y_{n} \subset(-2,2)$ be such that $y \in Y_{n}$ if and only if the $\tau_{Y}$-action on non-fixed points $(x, y, z) \in E_{\kappa}$ is periodic with period less than or equal to $n$. This gives a filtration

$$
\{0\}=Y_{2} \subset Y_{3} \subset \ldots \subset Y_{n} \subset \ldots
$$


For example: $Y_{2}=\{0\}, Y_{3}=\{0,1,-1\}, Y_{4}=\{0,1,-1, \sqrt{2},-\sqrt{2}\}$, etc. Note that the filtration is independent of the choice of $\kappa$ and that $Y_{n}$ is a finite set for every $n$. By symmetry, there are similar filtrations $X_{n}$ and $Z_{n}$, with $X_{n}=Y_{n}=Z_{n}$ as sets. The following lemmas, though proven for the $Y_{n}$ filtration, apply equally to the other filtrations.

Lemma 6.2. For $\epsilon>0$ there exists $N(\epsilon)>0$ so that if $y \notin Y_{N(\epsilon)}$, then the $\tau_{Y}$-orbit of $(x, y, z)$ is $\epsilon$-dense in $Y_{\kappa}(y)$ for any $(x, y, z)$ in any $E_{\kappa}$ (see part 7 of Definition 1.5).

Proof. Since the ellipses $Y_{\kappa}(y)$ are (possibly degenerate) of uniformly bounded circumferences, there exists $N(\epsilon)>1$ such that for any $y \notin Y_{N(\epsilon)}$, the $\tau_{Y}$-orbit is $\epsilon$-dense in $Y_{\kappa}(y)$.

Throughout the remainder of the paper, the moduli spaces of four-holed spheres with $\kappa=(a, b, c, d)$, having small $|c|$ and $|d|$, play an important role. We first analyze the case of $E_{\kappa}$ for $\kappa=(a, b, 0,0)$.

Lemma 6.3. Suppose $(a, b, 0,0)=\kappa \in(-2,2)^{4}$. Then $X_{\kappa}(x) \subset E_{\kappa}$ is an ellipse (possibly degenerate) centered at $(x, 0,0)$. Thus, $Y_{\kappa}(0)$ and $Z_{\kappa}(0)$ intersect every ellipse $X_{\kappa}(x)$.

Proof. This result follows directly from equation (1).

Lemma 6.4. Let $a, b \in(-2,2)$ and $\epsilon>0$. Then there exists $\delta>0$ so that for any $\kappa=(a, b, c, d)$ with $|c|,|d|<\delta$ the following hold:

1. The set

$$
\bigcap_{|y|<\delta}\left\{x:(x, y, z) \in Y_{\kappa}(y)\right\}
$$

is $\epsilon$-dense inside $\left\{x:(x, y, z) \in E_{\kappa}\right\}$, i.e., for all $|y|<\delta$, the set of $x$-coordinates of $Y_{\kappa}(y)$ is $\epsilon$-dense in the set of all x-coordinates of points in $E_{\kappa}$.

2. For all $x \in\left\{x:(x, y, z) \in E_{\kappa}\right\}$, either $\left\{y:(x, y, z) \in X_{\kappa}(x)\right\} \subset[-\delta, \delta]$ or $\left[-\frac{\delta}{2}, \frac{\delta}{2}\right] \subset\left\{y:(x, y, z) \in X_{\kappa}(x)\right\}$, i.e., the set of $y$-coordinates of any given $X_{\kappa}(x)$ either contains $\left[-\frac{\delta}{2}, \frac{\delta}{2}\right]$ or is contained in $[-\delta, \delta]$;

3. For all $x \in\left\{x:(x, y, z) \in E_{\kappa}\right\}$, either $\left\{z:(x, y, z) \in X_{\kappa}(x)\right\} \subset[-\delta, \delta]$ or $\left[-\frac{\delta}{2}, \frac{\delta}{2}\right] \subset\left\{z:(x, y, z) \in X_{\kappa}(x)\right\}$.

Proof. The result holds by the continuous dependence of $E_{\kappa}$ on $c$ and $d$, by the continuous dependence of $X_{\kappa}(x)$ on $x$, by the geometry of $E_{\kappa}$ for $\kappa=(a, b, 0,0)$ as described in Lemma 6.3 and by the fact that $a, b \neq \pm 2$.

\section{The Two-Holed Torus}

In this section, we prove Theorem 1.4 for the case of $g=1$ and $n=2$. The fundamental group $\pi_{1}(M, O)$ has a presentation

$$
\left\langle X, Y, A, B: X Y X^{-1} Y^{-1}=B A\right\rangle,
$$

where $A$ and $B$ represent the boundary components. Let

$$
K=X Y X^{-1} Y^{-1}, \quad W=A X, \quad W^{\prime}=X B, \quad Z=X Y
$$

(see Figure 3).

Cutting $M$ along $K$ yields a one-holed torus with boundary $K$ and a three-holed sphere with boundaries $A, B, K$. If $a= \pm 2$ (resp. $b= \pm 2$ ), then $A= \pm I$ (resp. 


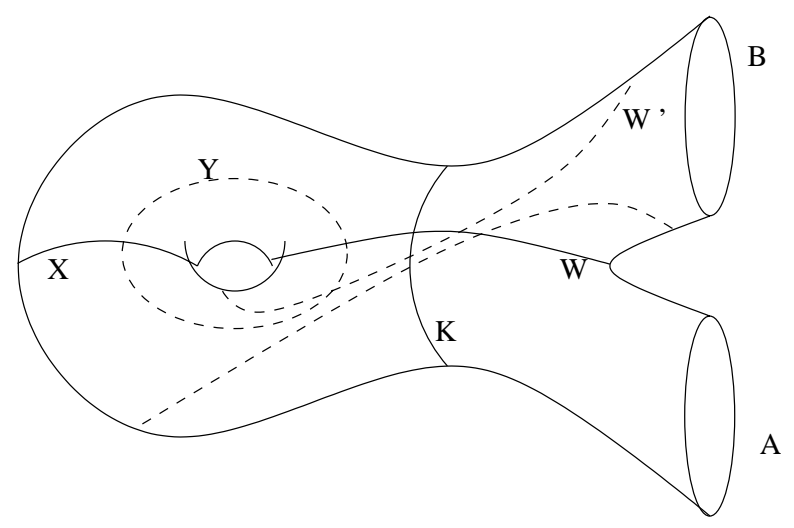

Figure 3. Two-holed torus.

$B= \pm I$ ). In this case the moduli space for $M$ can be identified with the moduli space of a one-holed torus (with $K=B A$ ), and so Theorem 1.3 applies. Thus we may assume that $a, b \neq \pm 2$.

Assume that $\rho \in \operatorname{Hom}\left(\pi_{1}(M, O), \mathrm{SU}(2)\right)$ is generic. By Proposition 3.1, we may assume without loss of generality that $\langle X, Y\rangle$ is a generic handle. If we cut $M$ along $X$ (resp. $Y$ ), then we obtain a four-holed sphere. For $\kappa=(b, a, x, x)$ (resp., $\kappa=(b, a, y, y))$, we obtain the moduli space $E_{\kappa}$ of a four-holed sphere.

The defining equation of the moduli space of this 4-holed sphere, $E_{\kappa}$, is

$$
\begin{gathered}
w^{2}+\left(w^{\prime}\right)^{2}+k^{2}+k w w^{\prime} \\
=k\left(a b+x^{2}\right)+w x(a+b)+w^{\prime} x(a+b)-a^{2}-b^{2}-2 x^{2}-a b x^{2}+4,
\end{gathered}
$$

and the formulas for the actions $\tau_{K}, \tau_{W}, \tau_{W^{\prime}}$ of the Dehn twists on the associated moduli space of this four-holed sphere become

$$
\begin{aligned}
& {\left[\begin{array}{c}
w \\
w^{\prime}
\end{array}\right] \stackrel{\tau_{K}}{\longmapsto}\left[\begin{array}{c}
x(a+b)-k\left(x(a+b)-k w-w^{\prime}\right)-w \\
x(a+b)-k w-w^{\prime}
\end{array}\right],} \\
& {\left[\begin{array}{c}
w^{\prime} \\
k
\end{array}\right] \stackrel{\tau_{W}}{\longmapsto}\left[\begin{array}{c}
x(a+b)-w\left(x^{2}+a b-w w^{\prime}-k\right)-w^{\prime} \\
x^{2}+a b-w w^{\prime}-k
\end{array}\right],} \\
& {\left[\begin{array}{c}
k \\
w
\end{array}\right] \stackrel{\tau_{W^{\prime}}}{\longmapsto}\left[\begin{array}{c}
a b+x^{2}-w^{\prime}\left(x(a+b)-w^{\prime} k-w\right)-k \\
x(a+b)-w^{\prime} k-w
\end{array}\right] .}
\end{aligned}
$$

Note that $I_{x, x}=\left[x^{2}-2,2\right]$ and that the actions $\tau_{W}$ and $\tau_{W^{\prime}}$ do not (in general) preserve $y$ or $z$ (see Figure 3), while the action $\tau_{K}$ preserves $x, y$, and $z$.

Lemma 7.1. For the representation $\rho$, there exists a $\gamma \in \Gamma$ such that at least one of the following has non-zero trace: $\gamma(A X), \gamma(X B), \gamma(A X Y), \gamma(X Y B), \gamma(A Y)$, $\gamma(Y B), \gamma(A Y X), \gamma(Y X B)$, with $\langle\gamma(X), \gamma(Y)\rangle$ generic.

Proof. Suppose that $A X, X B, A X Y, X Y B, A Y, Y B, A Y X$, and $Y X B$ all have zero trace. If $\tau_{K}$ preserves $w=w^{\prime}=0$, then $x(a+b)=0$ (if not, note that the twist in $\tau_{K}$ preserves $x, y$ and $k$, so $\left\langle\tau_{K}(X), \tau_{K}(Y)\right\rangle$ remains generic). By Remark [5.4 $x$ can be assumed non-zero. Hence $a=-b$. The equation for $E_{(-a, a, x, x)}$ is

$$
k^{2}+k a^{2}+2 a^{2}-4=x^{2}\left(k-2+a^{2}\right) .
$$


By Remark 5.4 and the fact that the left-hand side of equation (2) is invariant under $\left\langle\tau_{X}, \tau_{Y}\right\rangle$, it must be that $k-2-a^{2}=0$. The formula $\operatorname{tr}\left(A B^{-1}\right)+\operatorname{tr}(A B)=$ $\operatorname{tr}(A) \operatorname{tr}(B)$ (see [6, 8]) and the fact that $K=B A$ give $\operatorname{tr}\left(A B^{-1}\right)=-2$. This implies that $A=-B$ as matrices.

Suppose $A=-B$. Conjugating by an element in $\mathrm{SU}(2)$, we may assume that

$$
X=\left[\begin{array}{cc}
x_{1}+y_{1} i & 0 \\
0 & x_{1}-y_{1} i
\end{array}\right], \quad Y=\left[\begin{array}{cc}
x_{2}+y_{2} i & z_{2} \\
-z_{2} & x_{2}-y_{2} i
\end{array}\right]
$$

and

$$
A=\left[\begin{array}{cc}
a_{1}+b_{1} i & c_{1}+d_{1} i \\
-c_{1}+d_{1} i & a_{1}-b_{1} i
\end{array}\right] .
$$

The equations $\operatorname{tr}(A X)=0, \operatorname{tr}(A Y)=0, \operatorname{tr}(A X Y)=0, \operatorname{tr}(A Y X)=0$, together with the matrix equation $K=-A^{2}=X Y X^{-1} Y^{-1}$ and $\operatorname{det}(A)=\operatorname{det}(X)=\operatorname{det}(Y)=1$ lead to one of the following: $x_{1}=0, \pm 1, a_{1}=0$ or $a_{1}= \pm \sqrt{1-x_{1}^{2}}$. By Remark 5.4] we have $a_{1} \neq \sqrt{1-x_{1}^{2}}$ and $x_{1} \neq 0, \pm 1$. This leaves us with the case $a_{1}=0$, which implies that $K=I$, contradicting the fact that $\langle X, Y\rangle$ is generic.

Combining Proposition 5.2 and Lemma 7.1 one obtains

Corollary 7.2. For a generic $\rho$, we may assume that not all of $\langle A X, Y\rangle,\langle X B, Y\rangle$, $\langle A Y, X\rangle$, and $\langle Y B, X\rangle$ are $\operatorname{Pin}(2)$.

By Remark 5.3 and Corollary 7.2 we assume without loss of generality that the action $\tau_{Y}$ does not fix $w=\operatorname{tr}(A X)$.

Proposition 7.3. Suppose $g=1, n=2$, and $\rho$ is a generic representation. Then the $\Gamma$-orbit $\Gamma([\rho])$ is dense in $\mathcal{M}_{\mathcal{C}}$.

Proof. Let $\epsilon>0$. Let $\rho$ be a generic representation. By Proposition 3.1, $M$ has a generic handle $\langle X, Y\rangle$ (we adopt the notation presented in Figure 3). By Proposition 2.3 it is enough to show that for any $\left[\rho_{0}\right] \in \mathcal{M}_{\mathcal{P}}$, there exists $\gamma \in \Gamma$ such that $\mathrm{d}\left(\gamma([\rho]),\left[\rho_{0}\right]\right)<\epsilon$. Let $x_{0}=\operatorname{tr}\left(\rho_{0}(X)\right), k_{0}=\operatorname{tr}\left(\rho_{0}(K)\right), w_{0}=\operatorname{tr}\left(\rho_{0}(W)\right)$, etc.

Cutting along $K$ and $X$ gives a pants decomposition of $M$. Hence, by Corollary 2.4 we only need to show that there exists $\gamma \in \Gamma$ such that $\gamma([\rho])$ satisfies $\left|\gamma(k)-k_{0}\right|<\epsilon$ and $\left|\gamma(x)-x_{0}\right|<\epsilon$.

The strategy is summarized as follows: Obtain a $\tau_{Y}$-orbit with a sufficiently large number of points that have the following properties:

1. $x$-coordinates that are sufficiently close to zero so that $E_{(b, a, x, x)}$ (the moduli space of the four-holed sphere obtained by cutting $M$ at $X$ ) contains points with $k$-coordinates equal to $k_{0}$;

2. each of the points has sufficiently many points on its respective $\tau_{W}$-orbits (in $\left.E_{(b, a, x, x)}\right)$ so that one of these points can further be moved via $\tau_{K}$ and $\tau_{W}$ to get a point with $k$-coordinate within $\epsilon$ of $k_{0}$.

Along the way, there will be a certain finite list of values to avoid, e.g. $\mathcal{S}$, to guarantee that the handle $\langle X, Y\rangle$ remains generic.

One potential obstacle is the possibility that the points on the $\tau_{Y}$-orbit themselves have $\tau_{W}$-actions that fix $\left(k, w, w^{\prime}\right) \in E_{(b, a, x, x)}$. If this occurs, we will show that we can resort to the $\tau_{W^{\prime}}$-action. This amounts to showing that $\left\langle Y, W^{\prime}\right\rangle$ itself is not $\operatorname{Pin}(2)$ and that the corresponding $\tau_{W^{\prime}}$-actions will not fix $\left(k, w, w^{\prime}\right) \in E_{(b, a, x, x)}$. 
Lemma 7.4. Suppose that $x, y, w \neq 0$ and $\tau_{W}\left(k, w, w^{\prime}\right)=\left(k, w, w^{\prime}\right) \in E_{(b, a, x, x)}$. Then we have the following:

1. If $\left\langle W^{\prime}, Y\right\rangle$ is $\operatorname{Pin}(2)$, then

$$
2 k=a b+x^{2} .
$$

2. If $\tau_{W^{\prime}}\left(k, w, w^{\prime}\right)=\left(k, w, w^{\prime}\right) \in E_{(b, a, x, x)}$, then

$$
x^{2}=\frac{2 k-a b}{1-\left(\frac{a+b}{k+2}\right)^{2}} .
$$

Proof. Case 1: $\left\langle W^{\prime}, Y\right\rangle$ is $\operatorname{Pin}(2)$. This implies that $w^{\prime}=0$. Hence,

$$
2 k=a b+x^{2} .
$$

Case 2: Suppose that $\tau_{W^{\prime}}\left(k, w, w^{\prime}\right)=\left(k, w, w^{\prime}\right) \in E_{(b, a, x, x)}$. Then

$$
\left\{\begin{aligned}
2 k & =a b+x^{2}-w w^{\prime}, \\
2 w^{\prime} & =x(a+b)-w k, \\
2 w & =x(a+b)-w^{\prime} k .
\end{aligned}\right.
$$

This leads to $2\left(w^{\prime}-w\right)=k\left(w^{\prime}-w\right)$. Since $k \neq \pm 2$, we have $w=w^{\prime}=\frac{x(a+b)}{k+2}$. Moreover, since $w \neq 0$, we have $a \neq-b$. This implies that

$$
x^{2}\left[1-\left(\frac{a+b}{k+2}\right)^{2}\right]=2 k-a b .
$$

If $a+b= \pm(k+2)$ and $k=\frac{a b}{2}$, then either $a= \pm 2$ or $b= \pm 2$. This contradicts our assumptions on $a$ and $b$. So, we must have

$$
x^{2}=\frac{2 k-a b}{1-\left(\frac{a+b}{k+2}\right)^{2}} .
$$

Since $\langle X, Y\rangle$ is generic, Lemma 7.1 implies that we may readily find points with $\tau_{Y}$-orbits having coordinates that satisfy $w \neq 0$ and $y \neq 0$, while violating both equations (3) and (5).

The following lemma follows immediately from Remark 5.4 Corollary 7.2 and the fact that the $\tau_{X}$ and $\tau_{Y}$ actions fix $k$.

Lemma 7.5. For any integer $J>0$, there is $\gamma \in \Gamma$ such that the $\tau_{Y}$-orbit of $\gamma(\rho)$ has at least $J$ points satisfying the following conditions:

1. The $x$-coordinates of these $J$ points have $|x|$ small enough so that $k_{0} \pm \frac{\epsilon}{2} \epsilon$ $I_{a, b} \cap I_{x, x}$ and $|x| \leq \delta$, where $\delta$ is provided in Lemma 6.4 for $E_{(b, a, x, x)}$.

2. These $J$ points do not belong to the real subvarieties defined by equations (3), (5), $y=0$, and $w=0$.

Consider the handle $\langle Y, W\rangle$. Choose $J$ sufficiently large in Lemma 7.5 so that one of the $J$ points on the $\tau_{Y}$-orbit of $\gamma(\rho)$, denoted $\gamma_{1}(\rho)$, has a $\tau_{W}$-orbit (resp. $\tau_{W^{\prime} \text {-orbit) with at least }}$

$$
N\left(\frac{\delta}{N\left(\frac{\epsilon}{2}\right)+3}\right)+3
$$

points having distinct $k$-coordinates inside $E_{\left(b, a, \gamma_{1}(x), \gamma_{1}(x)\right)}$. 


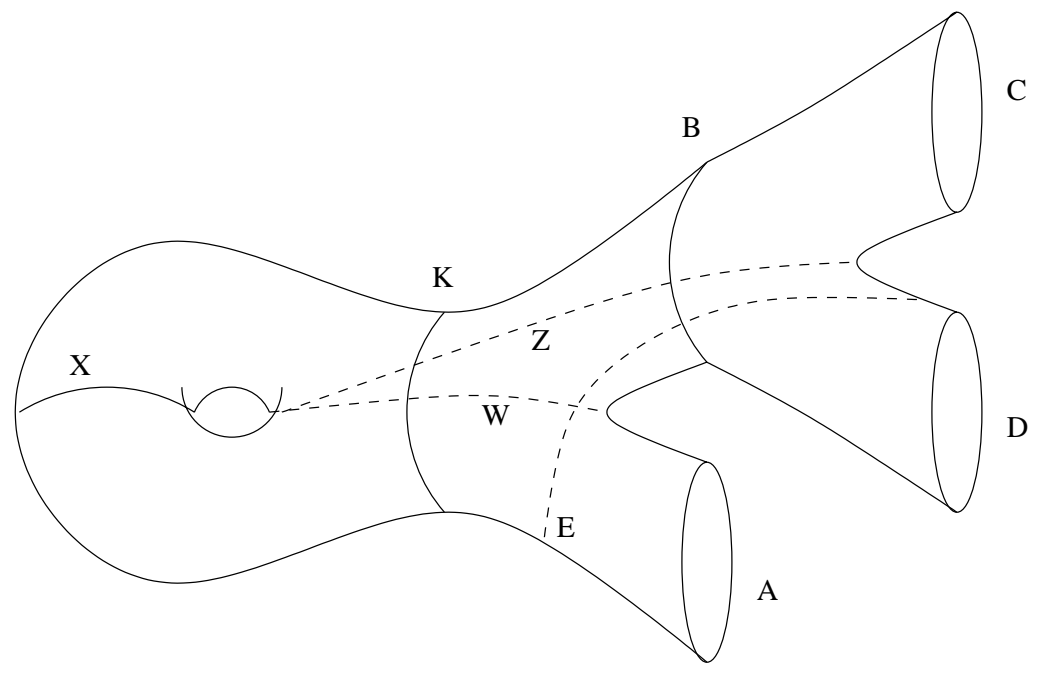

FiguRE 4. Three-holed torus.

Since $\left|\gamma_{1}(x)\right| \leq \delta$ and $k_{0} \pm \epsilon \in I_{a, b} \cap I_{\gamma_{1}(x), \gamma_{1}(x)}$, the sphere $E_{\kappa}$ (with $\kappa=$ $\left.\left(b, a, \gamma_{1}(x), \gamma_{1}(x)\right)\right)$ has points with $k$-coordinates inside $\left[k_{0}-\frac{\epsilon}{2}, k_{0}+\frac{\epsilon}{2}\right]$. Since the $\tau_{W}$-orbit (resp. $\tau_{W^{\prime}}$ ) of $\gamma_{1}(\rho)$ has at least $N\left(\frac{\delta}{N\left(\frac{\epsilon}{2}\right)+3}\right)+3$ points with distinct $k$ coordinates, One such point $\gamma_{2}(\rho)$, has $k$-coordinate not in $K_{N\left(\frac{\delta}{N\left(\frac{\delta}{2}\right)+3}\right)}$ (where $K_{n}$ denotes the filtration in $K$ ) with non-degenerate ellipse $K_{\kappa}\left(\gamma_{2}(k)\right) \subset E_{\kappa}$. Thus, the $\tau_{K}$ orbit of $\gamma_{2}(w)$ is $\frac{\delta}{N\left(\frac{\epsilon}{2}\right)+3}$-dense. The $\tau_{K}$-orbit of $\gamma_{2}(\rho)$ has at least $N\left(\frac{\epsilon}{2}\right)+3$ points with $w$-coordinates inside $(-\delta, \delta)$. One such point, $\gamma_{3}(\rho)$ has $w$-coordinate not in $W_{N\left(\frac{\epsilon}{2}\right)}$ with non-degenerate ellipse $W_{\kappa}\left(\gamma_{3}(w)\right)$. Thus, the $\tau_{W}$-orbit of $\gamma_{3}(\rho)$ is $\frac{\epsilon}{2}$-dense in $W_{\kappa}\left(\gamma_{3}(w)\right)$. This fact, together with the properties of $\delta$ provided in Lemma 6.4 implies that at least one point, $\gamma_{4}([\rho])$, in the $\tau_{W}$-orbit of $\gamma_{3}(\rho)$ has $k$-coordinate $\gamma_{4}(k)$ that comes within $\epsilon$ of $k_{0}$.

The restriction to the one-holed torus $\left\langle\gamma_{4}(X), \gamma_{4}(Y)\right\rangle$ is generic so long as $\gamma_{4}(k) \notin$ $\mathcal{S}$ and $\gamma_{4}(k) \neq\left(\gamma_{4}(x)\right)^{2}-2$ (see Proposition 5.2 and Remark [5.5). This can be accomplished by replacing $\epsilon$ by $\frac{\epsilon}{20}$ at the start of the argument. Since $\left\langle\gamma_{4}(X), \gamma_{4}(Y)\right\rangle$ is generic, we next apply $\tau_{X}$ and $\tau_{Y}$ to obtain $\gamma_{5} \in \Gamma$ so that the $x$-coordinate $\gamma_{5}(x)$ of $\gamma_{5}([\rho])$ is within $\epsilon$ of $x_{0}$. Note that both $\tau_{X}$ and $\tau_{Y}$ fix $\gamma_{4}(k)$. Thus $\left\langle\gamma_{5}(X), \gamma_{5}(Y)\right\rangle$ remains generic. The result now follows from Corollary 2.4

\section{The $n$-Holed Torus}

The conjugacy classes that correspond to $c_{i}= \pm 2$ are central. Thus, for $n>2$, one may assume that $C_{i} \neq \pm I$ for all $i$, or that $n=1$ and $C_{1}= \pm I$.

Proposition 8.1. Let $M$ be an $n$-holed torus and $\rho$ a generic representation with generic handle $\langle X, Y\rangle$, which is part of a pants decomposition $\mathcal{P}$, i.e., both $K=$ $X Y X^{-1} Y^{-1} \in \mathcal{P}$ and $X \in \mathcal{P}$. Then there is $\gamma \in \Gamma$ so that $\gamma(P) \neq \pm I$ for all $P \in \mathcal{P}$. Moreover, the handle $\langle\gamma(X), \gamma(Y)\rangle$ is generic.

Proof. We first treat the case of $n=3$. Let $C$ and $D$ be two boundary loops separated from $\langle X, Y\rangle$ by $B=C D$. Let $A$ be the remaining boundary loop (see 
Figure 4). Since $\langle X, Y\rangle$ is generic, we have that $K, X \neq \pm I$. The goal, therefore, is to find $\gamma \in \Gamma$ such that $\gamma(B) \neq \pm I$.

Suppose $B= \pm I$. Then $C= \pm D^{-1}$ and $A= \pm K^{-1}$. If $A D=E \neq \pm I$, then we may apply Proposition 7.3 to the two-holed torus bounded by $E$ and $C$. Hence, if $E \neq \pm I$, there exists an element $\gamma \in \Gamma$ that fixes $a$ with $\gamma(k) \neq \pm a$ such that $\langle\gamma(X), \gamma(Y)\rangle$ is generic. This implies $\gamma(B) \neq \pm I$. The same conclusion can be drawn if $A C \neq \pm I$. Thus, we assume that $A C= \pm I$ and $A D= \pm I$. Since $A= \pm K^{-1}$, we have that $C, D= \pm K$. Hence, $B=C D= \pm K^{2}= \pm I$. This is only possible if $k=0$ or $K= \pm I$. The latter case is ruled out by the generic assumption on $\langle X, Y\rangle$. Hence $k=0$. We will show that in this special case, we can obtain $\gamma(B) \neq \pm I$.

Suppose that $X$ commutes with $K$. If $\tau_{Y}(X)=X Y$ also commutes with $K$, then $X, Y$, and $K$ all belong to the same one-parameter subgroup of $\mathrm{SU}(2)$. Hence $X$ and $Y$ commute, contradicting the fact that $\langle X, Y\rangle$ is generic. Suppose that $X K X^{-1} K^{-1}=-I$ ( $X$ and $K$ anti-commute). Then, $X=K(-X) K^{-1}$, which implies that $x=0$. Again, since $\langle X, Y\rangle$ is generic, we may assume that $x \neq 0$. To summarize, since $\langle X, Y\rangle$ is generic, one may arrange that $X$ neither commutes nor anti-commutes with $K$ (i.e. $X K X^{-1} K^{-1} \neq \pm I$ ).

Now consider the curve $Z=X C$. The Dehn twist in $Z$ preserves $E= \pm I$ and $c$. Hence it fixes $k=0$ (consider the pants bounded by $C, E$, and $K$ ). On the four-holed sphere bounded by $C, D, X$ and $W$, the action of $\tau_{Z}$ on the matrix $B$ is $\tau_{Z}(B)=C Z D Z^{-1}= \pm C X C D C^{-1} X^{-1}$. Since $C, D= \pm K$ and $K^{2}=$ $-I, \tau_{Z}(B)= \pm K X K^{-1} X^{-1} \neq \pm I$. Hence there exists $\gamma \in \Gamma$ with $\gamma(B) \neq \pm I$. By Remark 5.4 we may assume that $x$ is not in $\left\{0, \pm 1, \pm \frac{1 \pm \sqrt{5}}{2}, \pm \sqrt{2}\right\}$. Since $\tau_{Z}$ preserves $k$ and $x$, the handle $\left\langle\tau_{Z}(X), \tau_{Z}(Y)\right\rangle$ is generic, as $k=0$ and $x \notin\{ \pm \sqrt{2}, 0\}$. This proves the case $n=3$.

The above argument may be repeated iteratively, starting with loops in $\mathcal{P}$ that bound two boundary loops and working inward towards $K$. We demonstrate using the case $n=5$, with notation provided in Figure 5 .

First consider the three-holed torus bounded by $E, F$, and $H=A C D$. Since $\langle X, Y\rangle$ is generic, if $B_{1}= \pm I$, then $A C D \neq \pm I$ (otherwise $K= \pm I$ ). Use the previous argument for $n=3$ to arrange $B_{1} \neq \pm I$. Next, the three-holed torus bounded by $B_{1}, D$, and $A C$ is used to make $B_{2} \neq \pm I$, etc.

Proposition 8.2. Suppose that $g=1, n=3$ and $\rho$ is a generic representation. Then the $\Gamma$-orbit $\Gamma([\rho])$ is dense in $\mathcal{M}_{\mathcal{C}}$.

Proof. The proof is similar in nature to the proof of Proposition 7.3 Consider the notation suggested in Figure 4.

Cutting along $X, K$ and $B$ yields a pants decomposition. Hence, by Proposition 7.3 and Corollary 2.4 we need to show that there exists $\gamma \in \Gamma$ such that $\gamma([\rho])$ satisfies $\left|\gamma(b)-b_{0}\right|<\epsilon$, with $\langle\gamma(X), \gamma(Y)\rangle$ generic. We first move $x$ and $w$ near zero so that the moduli space of the four-holed sphere bounded by $X, W, C$, and $D$ contains $b$-coordinates near $b_{0}$. We use Propositions 8.1 and 7.3 to do this.

As in the proof of Proposition 7.3, we wish to obtain a $\tau_{Y}$-orbit with a sufficiently large number of points that have the following properties:

1. $x$ - and $w$-coordinates that are sufficiently near zero so that the moduli space of the four-holed sphere bounded by $X, W, C$, and $D$ contains points with $b$-coordinate equal to $b_{0}$; 
2. each of these points has sufficiently many points on their respective $\tau_{Z}$-orbits (in $E_{(x, w, c, d)}$ ) so that one such point can further be moved via $\tau_{B}$ and $\tau_{Z}$ on $E_{(x, w, c, d)}$ to get a point with a $b$-coordinate within $\epsilon$ of $b_{0}$.

For this strategy to work, we must first have that $\langle Y, Z\rangle$ or $\left\langle Y, Z^{\prime}\right\rangle$ is not $\operatorname{Pin}(2)$, where $Z^{\prime}=D X$. If both $\langle Y, Z\rangle$ and $\left\langle Y, Z^{\prime}\right\rangle$ are $\operatorname{Pin}(2)$, then $z=z^{\prime}=0$ and $\operatorname{tr}\left(Y Z^{\prime}\right)=\operatorname{tr}(Y Z)=0$. Note that the application of $\tau_{B}$ fixes the moduli space of the two-holed torus bounded by $A$ and $B$, i.e., $\tau_{B}$ fixes $a, b, x, y, k$, and $w$. If the Dehn twist $\tau_{B}$ fixes $\left(b, z, z^{\prime}\right)$ with $z=z^{\prime}=0$, then we have

$$
w d+x c=0
$$

and

$$
x d+w c=0 .
$$

In the special case where $c=d=0$, the defining equation for $E_{(x, w, c, d)}$ yields

$$
b^{2}=x w b-x^{2}-w^{2}+4 .
$$

However, by Proposition 7.3 and the fact that $b, c, d$ are fixed by mapping class actions on the two-holed torus bounded by $B$ and $A$, we may assume that the $x, w$ coordinates do not satisfy any of the above equations. Hence, we may assume that $\langle Y, Z\rangle$ is not $\operatorname{Pin}(2)$.

We consider the possibility that the points on the $\tau_{Y^{-}}$-orbit themselves have $\tau_{Z^{-}}$ actions that fix $\left(b, z, z^{\prime}\right) \in E_{(x, w, c, d)}$. As before, in such cases we will rely on the $\tau_{Z^{\prime}}$-action.

If $\tau_{Z}$ fixes $\left(b, z, z^{\prime}\right) \in E_{(x, w, c, d)}$ and $\left\langle Y, Z^{\prime}\right\rangle$ is $\operatorname{Pin}(2)$, then

$$
2 b=x w+c d .
$$

If neither $\langle Y, Z\rangle$ nor $\left\langle Y, Z^{\prime}\right\rangle$ is $\operatorname{Pin}(2)$ but both $\tau_{Z}$ and $\tau_{Z^{\prime}}$ fix $\left(b, z, z^{\prime}\right)$, then

$$
\left\{\begin{array}{l}
2 b=x w+c d-z z^{\prime}, \\
2 z=x c+w d-b z, \\
2 z^{\prime}=x d+w c-b z^{\prime} .
\end{array}\right.
$$

This implies that $\tau_{B}$ also fixes $\left(b, z, z^{\prime}\right)$. As neither $\langle Y, Z\rangle$ nor $\left\langle Y, Z^{\prime}\right\rangle$ is $\operatorname{Pin}(2)$, we may assume that $z, z^{\prime} \neq \pm 2$. Hence $B_{\kappa}(b), Z_{\kappa}(z)$ and $Z_{\kappa}^{\prime}\left(z^{\prime}\right)$ are single points. This leads to $I_{x, w} \cap I_{c, d}=\{b\}$. However, as $b \neq \pm 2$, one can use Proposition [7.3 to guarantee that the $x, w$-coordinates on sufficiently many points of a $\tau_{Y}$-orbit do not satisfy equation (91) and are close enough to zero so that $b$ is not an endpoint of $I_{x, w}$.

The argument now follows similarly to the one presented in Proposition 7.3 to produce $\gamma \in \Gamma$ satisfying $\left|\gamma(b)-b_{0}\right|<\epsilon$.

Proposition 8.3. Suppose that $M$ is a genus one surface with $n \geq 1$ boundary components. Let $\rho$ be a generic representation. Then the $\Gamma$-orbit $\Gamma([\rho])$ is dense in $\mathcal{M}_{\mathcal{C}}$.

Proof. The cases $n=1,2$, and 3 have previously been established. For $n \geq 4$, the argument follows by an induction process similar to that in the proof of Proposition 8.1 


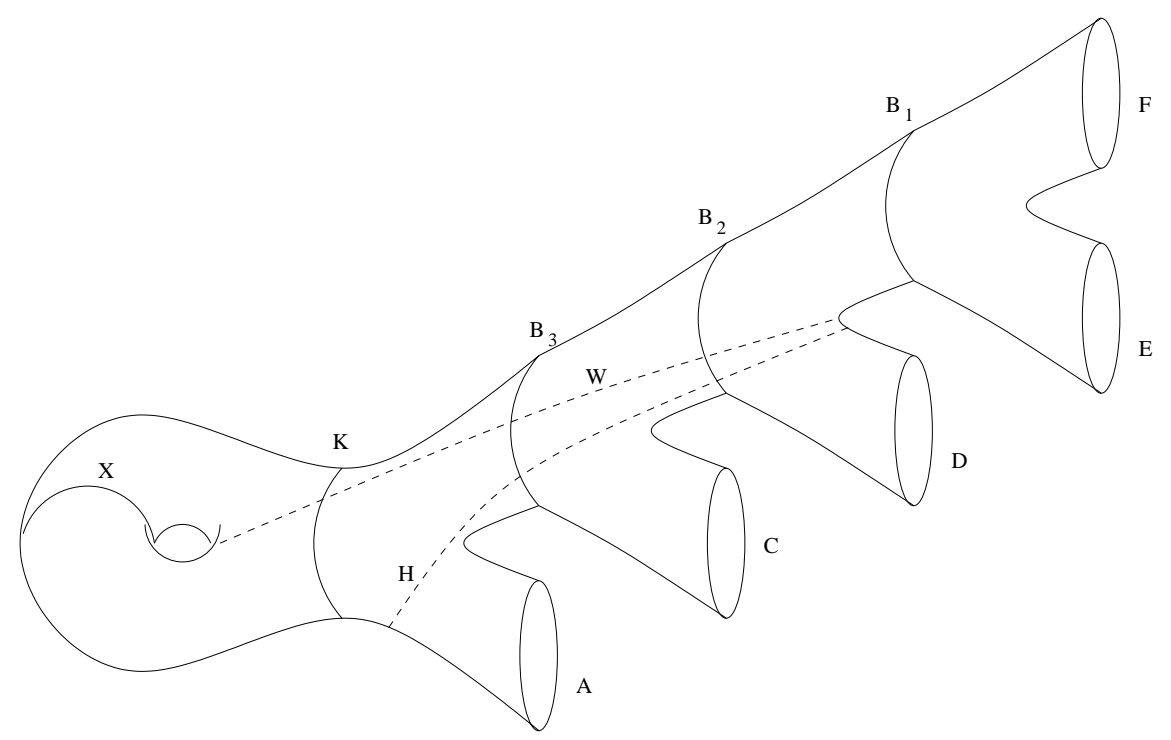

FiguRE 5. Getting rid of $\pm I$ on $\mathcal{P}$ for $n=5$.

Let $\rho$ be a generic representation. By Proposition 3.1, $M$ has a generic handle $\langle X, Y\rangle$. We demonstrate how to proceed in the case $n=5$ (see Figure 5). Let $\left[\rho_{0}\right] \in \mathcal{M}_{\mathcal{P}}$.

By Proposition 8.1, we have that $B_{1} \neq \pm I$. Assume that Proposition 8.3 is true for the four-holed torus bounded by $B_{1}, A, C$ and $D$. We use this four-holed torus to arrange for $x$ and $w$ (see Figure 5 ) to have near zero traces. This ensures that the $b_{1}$-coordinate of $\left[\rho_{0}\right]$ is accessible. We then arrange $H \neq \pm I$. Next, cut $M$ at $H$ and use Proposition 8.2 to get the $b_{1}$-coordinate of $\gamma([\rho])$ within $\epsilon$ of $\left(b_{1}\right)_{0}$. Next, we get the $b_{2}$-coordinate of $\gamma([\rho])$ within $\epsilon$ of $\left(b_{2}\right)_{0}$, by using the four-holed torus obtained by cutting at $B_{1}$. Finally, Proposition 8.2 and Corollary 2.4 yield the result. The situation is identical for any $n>3$.

\section{Genus $g$ with $n$ Boundary Components}

Suppose $M$ is a surface with $g>1$ and $n \geq 0$. Again, we assume that $C_{i} \neq \pm I$ for all boundary curves $C_{i} \in \partial M$, unless $n=1$ and $C_{1}= \pm I$.

Proposition 9.1. Let $M$ have generic $\langle X, Y\rangle$ that is part of a pants decomposition $\mathcal{P}$. Then there is $\gamma \in \Gamma$ so that $\gamma(\rho(P)) \neq \pm I$, for all $P \in \mathcal{P}$ with $\langle\gamma(X), \gamma(Y)\rangle$ generic. (In the special case $n=1$ and $C_{1}= \pm I$, we have $\gamma(\rho(P)) \neq \pm I$ for all $P \in \mathcal{P}$ except $C_{1}$.) Furthermore, $\langle\gamma(P), \gamma(Q)\rangle$ is generic, for all $P \in \mathcal{P}$ and $P Q P^{-1} Q^{-1}=R \in \mathcal{P}$ that bound a three-holed sphere which forms a one-holed torus in $M$.

Proof. We first show that there is $\gamma \in \Gamma$ so that $\langle\gamma(P), \gamma(Q)\rangle$ is generic for all $P \in \mathcal{P}$ and $P Q P^{-1} Q^{-1}=R \in \mathcal{P}$ that bound a three-holed sphere which forms a one-holed torus in $M$. Consider $P, Q$, and $R$ as in Figure 6 , where $P, R \in \mathcal{P}$ bound a pants that forms a one-holed torus in $M$.

Case 1: Suppose that $R \neq \pm I$. In this case, at least one of $p, q$ or $\operatorname{tr}(P Q)$ is not in $\{0, \pm 2\}$. Without loss of generality, assume that $p \notin\{0, \pm 2\}$. Thus, we 


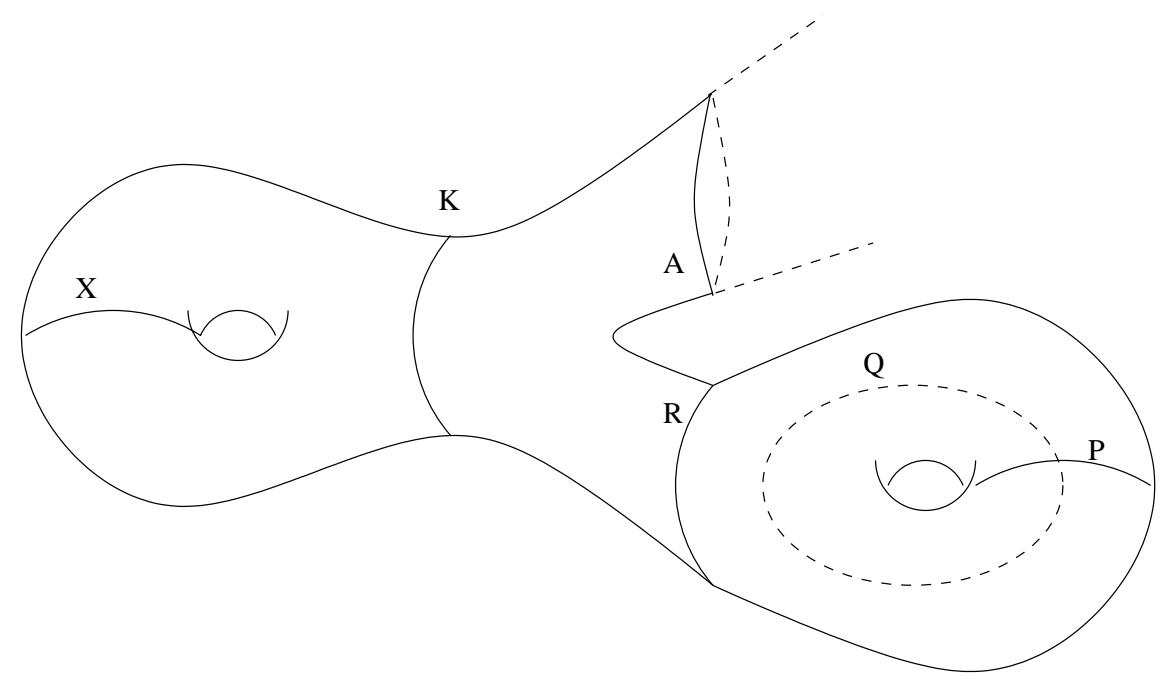

Figure 6. A punctured genus two inside $M$.

may cut $M$ at $P$ and $A$ and apply Proposition 8.2 to the resulting three-holed torus to obtain $r \notin \mathcal{S}$ with $r \neq p^{2}+2$. Note that in the special case $A= \pm I$, the corresponding three-holed torus can be identified to that of a two-holed torus, to which Proposition 7.3 can be applied. Thus the handle $\langle\gamma(P), \gamma(Q)\rangle$ is generic.

Case 2: Suppose that $R=-I$. Then both $P$ and $Q$ are not $\pm I$. Moreover, $A \neq \pm I$ since $K \neq \pm I$. Thus, we may cut $M$ at $P$ and $A$, and then apply Proposition 8.2 to reduce the situation to Case 1 .

Case 3: Suppose that $R=I$. Then, necessarily, $A= \pm K^{-1} \neq \pm I$, since $\langle X, Y\rangle$ is generic. If either $P \neq \pm I$ or $Q \neq \pm I$, we may cut at $P$ (or $Q$ ) and apply Proposition 8.2 as in case 2. If both $P= \pm I$ and $Q= \pm I$, then $\tau_{P A}(Q)=$ $Q(P A)= \pm K \neq \pm I$. Note that the action of $\tau_{P A}$ does not affect the generic handle $\langle X, Y\rangle$. We now cut at $Q$ and apply Proposition 8.2 as in case 2. This argument can be applied independently to each of the $g-1$ handles of $M$.

Having obtained that all handles are generic, we now apply Proposition 8.1 to the remaining curves in $\mathcal{P}$ that are interior to the $(n+g-1)$-holed torus obtained by cutting off each of the $g-1$ handles. In the special case $n=1$ and $C_{1}= \pm I$, Proposition 8.1 applies to those curves of $\mathcal{P}$ that are separated from $C_{1}$ by the curve $K C_{1}$. Moreover, $K C_{1} \neq \pm I$ since $\langle X, Y\rangle$ is assumed generic.

Now we prove Theorem 1.4 Let $\rho$ be a generic representation. By Proposition 3.1, $M$ has a generic handle $\langle X, Y\rangle$ (we adopt the notation presented in Figure 7). By Proposition 2.3, without loss of generality we may take $\left[\rho_{0}\right] \in \mathcal{M}_{\mathcal{P}}$ and show that there exists $\gamma \in \Gamma$ such that $\mathrm{d}\left(\gamma([\rho]),\left[\rho_{0}\right]\right)<\epsilon$.

By Proposition 9.1, we may assume that $\left\langle P_{i}, Q_{i}\right\rangle$ is generic and that $p_{i} \notin\{0, \pm 2\}$ for all $1 \leq i \leq g-1$.

Dehn twist each generic handle $\left\langle P_{i}, Q_{i}\right\rangle$ to obtain $p_{i}$ arbitrarily close to zero. Since $x$ and $z_{i}=\operatorname{tr}\left(R_{i} X\right)$ can be made arbitrarily close to zero by Proposition 8.3 (one can do this also in the exceptional case $n=1, C_{1}= \pm I$, and $g>1$ ), we are ensured that the target value $r_{i}$-coordinate of $\rho_{0}$ will be inside the $\epsilon$-neighborhood 


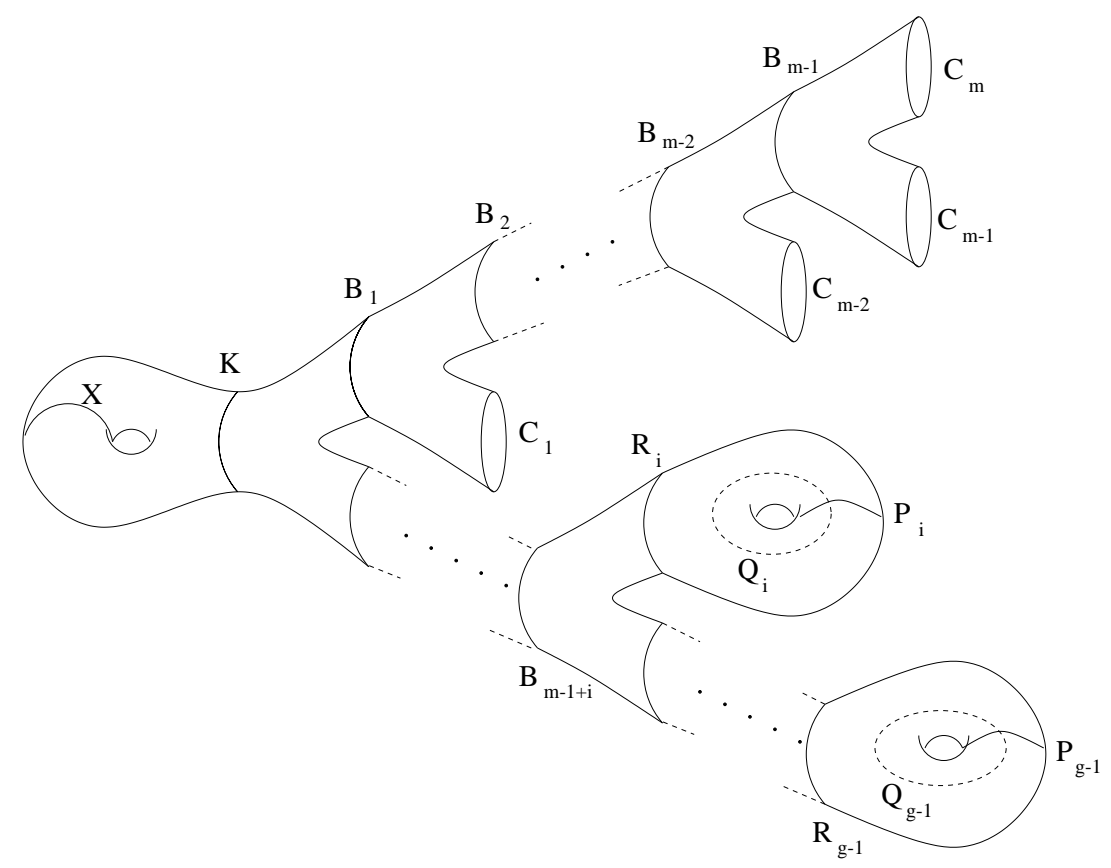

FiguRE 7. Decomposition of $M$.

of $I_{p_{i}, p_{i}} \cap I_{x, z_{i}}$. Thus, there are points on the moduli space of the four-holed sphere $E_{\left(p_{i}, p_{i}, x, z_{i}\right)}$ with $r_{i}$-coordinates within $\epsilon$ of $\left(r_{i}\right)_{0}$, the $r_{i}$-coordinate of $\rho_{0}$.

We next apply Proposition 8.3 to the $(n+2 g-2)$-holed torus obtained by cutting $M$ at each of the $P_{i}$ to move each $r_{i}$ within $\epsilon$ of $\left(r_{i}\right)_{0}$, keeping $\left\langle\gamma\left(P_{i}\right), \gamma\left(Q_{i}\right)\right\rangle$ generic. Again, this is also possible in the special case of $n=1, C_{1}= \pm I$, and $g>1$, as the moduli space of the $(1+2 g-2)$-holed torus reduces to that of a generic $(2 g-2)$-holed torus.

Next, we twist on each of the $g-1$ generic handles to get $p_{i}$ within $\epsilon$ of $\left(p_{i}\right)_{0}$. Finally, we cut off each handle at $R_{i}$ and apply Proposition 8.3 to the resulting $(n+g-1)$-holed torus with generic $\langle\gamma(X), \gamma(Y)\rangle$.

Theorem 1.4 now follows from Corollary 2.4

\section{REFERENCES}

[1] Benedetto, R. L., and Goldman, W. M., The topology of the relative character varieties of a quadruply-punctured sphere Experimental Mathematics, 8 (1) (1999), 85-104. MR 2000c:57028

[2] Conway, J. H. and Jones, A. J., Trigonometric diophantine equations (On vanishing sums of roots of unity) Acta Arithmetica, Vol. XXX, (1976), 229-240. MR 54:10141

[3] Gallo, D., Kapovich, M., Marden, A., The Monodromy groups of Schwarzian equations on closed Riemann surfaces, Ann. of Math. (2) 151 (2000), 625-704.

[4] Goldman, W. M., Ergodic theory on moduli spaces Ann. of Math., Vol. 146, (1997), 475-507. MR 99a:58024

[5] Goldman, W. M., The symplectic nature of fundamental groups of surfaces, Adv. Math., Vol. 54, (1984), 200-225. MR 86i:32042

[6] Horowitz, R., Characters of free groups represented in the two-dimensional special linear group, Comm. Pure Appl. Math., Vol. 25, (1972), 635-649. MR 47:4542 
[7] Jeffrey, L., Weitsman, J., Bohr-Sommerfeld orbits in the moduli space of flat connections and the Verlinde dimension formula, Comm. Math. Phys., Vol. 150, (1992), 595-630. MR 94g:58085

[8] Magnus, W., Rings of Fricke characters and automorphism groups of free groups, Mathematische Zeitschrift, Vol. 170, (1980), 91-103. MR 81a:20043

[9] Previte, J. P., Xia, E. Z., Topological dynamics on moduli spaces I, Pacific Journal of Mathematics Vol 193, No 2, (2000), 397-418. MR 2001e:53090

[10] Seshadri, C., Fibrés vectoriels sur les courbes algébriques, Astérisque, 96 (1982). MR 85b:14023

School of Science, Penn State University Erie, The Behrend College, Erie, PennSYLVANIA 16563

E-mail address: jpp@vortex.bd.psu.edu

Department of Mathematics \& Statistics, University of Massachusetts, Amherst, MASSACHUSETTS 01003-4515

E-mail address: xia@math.umass.edu 